\title{
INTEGRAL FORMULAS RELATED TO WAVE FRONTS
}

\author{
SERGEI S. ANISOV \\ Independent University of Moscow \\ Bol'shoŭ Vlas'evskiu 11, 121002 Moscow, Russia \\ and Institute for System Studies \\ E-mail: anisov@mccme.ru
}

\begin{abstract}
In the first section of the paper we study some properties of oriented volumes of wave fronts propagating in spaces of constant curvature. In the second section, we generalize to an arbitrary isometric action of a Lie group on a Riemannian manifold the following principle: an extra pression inside of a ball does not move it.
\end{abstract}

1. Wave fronts in $S^{n}$ and $H^{n}$. The standard sphere $S^{n} \subset \mathbb{R}^{n+1}$ is defined by the equation $x_{0}^{2}+x_{1}^{2}+\ldots+x_{n}^{2}=1$. The metric on the sphere is induced by the standard metric in $\mathbb{R}^{n+1}$. Thus the distance between points $X, Y$ of the sphere is equal to the angle $X O Y$, where $O$ stands for the center of the sphere. Let $F \subset S^{n}$ be a cooriented wave front, which is a projection of an oriented Legendrian manifold. Let $F$ move in $S^{n}$ with unit velocity in the direction of the coorientation. Denote by $F(t)$ the position of the front at a moment $t$ and by $c(t)$ the oriented $(n-1)$-dimensional volume of $F(t)$. Obviously, $F(t+2 \pi)=F(t)$, and $F(t+\pi)$ is nothing but the front centrally symmetric to $F(t)$, whereas $F(t+\pi / 2)$ is the front dual to $F(t)$ [2]. Thus $c(t+2 \pi)=c(t), c(t+\pi)=-c(t)$ for $n$ even, and $c(t+\pi)=c(t)$ for $n$ odd. For $n$ even, let $s(t)$ be a primitive function for $c(t)$. Then $s(t+2 \pi)=s(t)$, because $c(t)$ is an odd $2 \pi$-periodic function. So $s(t)$ can be regarded as the volume bounded by $F(t)$; this is motivated by the property $s^{\prime}(t)=c(t)$. To make the definition unambiguous, impose the condition $s(0)+s(\pi)=0$, which implies that $s$ is an odd function: $s(t+\pi)=-s(t)$ for any $t$.

Let us recall some results on wave fronts in $S^{2}$.

1991 Mathematics Subject Classification: Primary: 51M25. Secondary: 51M04, 51M09, 53A17, 53C65.

This research was supported by the Russian Foundation for Basic Research under grant No. 95-01-00846 and by CEREMADE, Université Paris-Dauphine.

The paper is in final form and no version of it will be published elsewhere. 
TheOREM 1 [4]. The length (respectively, the area) of a front in $S^{2}$ is equal up to a sign to the area (respectively, length) of its dual front. More precisely,

$$
s\left(t+\frac{\pi}{2}\right)=c(t) \quad \text { and } \quad c\left(t+\frac{\pi}{2}\right)=-s(t)
$$

The paper [1] contains a proof of this theorem based on the following idea. Since $s(t)$ and $c(t)$ are odd $2 \pi$-periodic functions satisfying the differential equations $s^{\prime}(t)=c(t)$ and $c^{\prime}(t)=-s(t)$ (the latter equation can be derived from the Gauss-Bonnet theorem), it is clear that $c(t)=a \cos (t+b)$ and $s(t)=a \sin (t+b)$ for some $a$ and $b$, and the theorem follows. Below we study the function $c(t)$ constructed from a front in $S^{n}$ or $H^{n}$, where $H^{n}$ is the $n$-dimensional Lobachevskii space. We consider only closed wave fronts, which are projections of smooth compact Legendrian submanifolds $\widetilde{F}$ of the contact manifold $P T^{*} S^{n}$, respectively $P T^{*} H^{n}$.

Theorem 2. The volume $c(t)$ of a front $F(t)$ moving in $S^{n}$ is a polynomial in $\sin t$ and $\cos t$ of degree $n-1$.

Proof. Let $\widetilde{F}$ be a Legendrian submanifold of $P T^{*} S^{n}$ such that $p(\widetilde{F})=F$, where $p: P T^{*} S^{n} \rightarrow S^{n}$ stands for the natural projection. For $x \in \widetilde{F}$, put $x(0)=p(x) \in F(0)$ and denote by $x(t)$ the point of $F(t)$ obtained from $x(0)$ by a shift by $t$ along the geodesic line directed by $\mathbf{n}$, where $\mathbf{n}$ is the normal vector to $F(0)$ at $x_{0}$ that defines the coorientation of the front. Let $d \sigma(x, t)$ be the volume element on $F(t)$, and let $k_{1}(x, t), \ldots, k_{n-1}(x, t)$ be the principal curvatures of $F(t)$ at $x(t)$. Let us define numbers $r_{i}(x, t)$ by the relations $k_{i}(x, t)=\cot r_{i}(x, t)$, where $i=1, \ldots, n-1$; these numbers are equal to the oriented distances in $S^{n}$ between $x(t)$ and the curvature center of the section of $F(t)$ by the twodimensional geodesic sphere containing $\mathbf{n}(x(t))$ and the $i$ th principal direction; of course, we deal here with the geodesic curvature center, which belongs to the same sphere. More precisely, the number $r_{i}(\bmod 2 \pi)$ is defined as follows: the geodesic lines directed by the normal vectors $\mathbf{n}(x)$ and $\mathbf{n}\left(x^{\prime}\right)$ to $F$ at infinitely close points $x$ and $x^{\prime}$ (such that the direction from $x$ to $x^{\prime}$ is the $i$ th principal direction) intersect one another at two antipodal points of the sphere $N_{i}(x, t)$ and $-N_{i}(x, t)$. Choose arbitrarily one of them, say, $N_{i}(x, t)$, continuously in $x$ and $t$; there are no obstructions to this choice, because we do this for a small part of $F$ only. Then $r_{i}(x, t)$ is the length of the arc of the great circle passing through $x(t)$ and $N_{i}(x, t)$ that is oriented from $N_{i}(x, t)$ to $x(t)$ by the coorientating vector. Denote by $d l_{i}(x, t)$ the length element of the $i$ th section. Since the principal directions are pairwise orthogonal, we have

$$
d \sigma(x, t)=d l_{1}(x, t) d l_{2}(x, t) \cdots d l_{n-1}(x, t) .
$$

Choose the metric on $\widetilde{F}$ so that the distance between contact elements $x$ and $y$ is the angle between the hyperplanes in $\mathbb{R}^{n+1}$ that pass through the origin $O$ and the point $p(x)$, respectively, $p(y)$, at which the element $x$ (respectively, $y$ ) is attached; further, these hyperplanes contain the $(n-1)$-dimensional plane defined by the element $x$, respectively, $y$. It is easy to see that this metric is defined by a smooth metric tensor $g_{i j}$ and thus determines the volume form $\mu$ on $\widetilde{F}$. Let $d l_{i}(x)$ be the length element on $\widetilde{F}$ corresponding to the $i$ th principal direction at $x(t) \in F(t)$. It can easily be checked that the relations $d l_{i}(x, t)=\sin r_{i}(x, t) d l_{i}(x)$ hold up to a sign, and that the directions of $d l_{i}(x)$ are pairwise 
orthogonal in $\widetilde{F}$. Thus we have

$$
d \mu(x)=d l_{1}(x) d l_{2}(x) \cdots d l_{n-1}(x) .
$$

Then the following relation holds up to a sign:

$$
d \sigma(x, t)=\sin r_{1}(x, t) \sin r_{2}(x, t) \cdots \sin r_{n-1}(x, t) d \mu(x) ;
$$

the minus sign can be eliminated by replacing one of the $N_{i}(x, t)$ by its antipodal point (then the corresponding distance $r_{i}$ will be replaced by $r_{i}+\pi$ ).

Let us show that

$$
r_{i}(x, t)=t+\psi(x),
$$

where $\psi(x)$ does not depend on $t$. Indeed, let us consider the mapping

$$
f: F(t) \times S^{1} \rightarrow S^{n}
$$

that takes $(x, t, \varphi)$ to the point obtained by shifting $x \in F(t)$ by $\varphi$ along the geodesic line directed by the coorientation. Then the set $N_{i}(x, t), i=1,2, \ldots, n$, consists of the images of critical points of mapping (4) belonging to $x \times S^{1}$ (see [6]); they are called the focal points. Also note that

$$
f(x, t, \varphi)=f(x, 0, \varphi+t) .
$$

So for different $t$ the sets of focal points of $F(t)$ are the same, which implies (3).

It follows from (2) and (3) that

$$
d \sigma(x, t)=\left(\sum_{k=0}^{n-1} a_{k}(x) \cos k t+\sum_{k=1}^{n-1} b_{k}(x) \sin k t\right) d \mu(x) .
$$

Integrating this relation over $F(t)$, we obtain

$$
\begin{aligned}
& c(t)=\operatorname{vol} F(t)=\int_{F(t)} 1 d \sigma(x, t) \\
&=\int_{\widetilde{F}}\left(\sum_{k=0}^{n-1} a_{k}(x) \cos k t+\sum_{k=1}^{n-1} b_{k}(x) \sin k t\right) d \mu(x) \\
&=\sum_{k=0}^{n-1} A_{k} \cos k t+\sum_{k=1}^{n-1} B_{k} \sin k t,
\end{aligned}
$$

where $A_{k}=\int_{\widetilde{F}} a_{k}(x) d \mu(x)$ and $B_{k}=\int_{\widetilde{F}} b_{k}(x)$. The theorem is proved

COROLLARY. Let $n$ be an even number. Then the volume $s(t)$ of the domain bounded by a front $F(t)$ (which moves in $S^{n}$ with unit velocity) is a trigonometric polynomial of degree $n-1$. The polynomials $s(t)$ and $c(t)$ are odd, that is, they do not contain terms $\sin k x$ and $\cos k x$ with $k$ even.

THEOREM 3. The oriented $(n-1)$-dimensional volume $c(t)$ of a front $F(t)$ moving in Euclidean space $\mathbb{R}^{n}$ with unit velocity is a polynomial in $t$ of degree $n-1$.

Proof. The proof of this statement is quite similar to that of Theorem 2. 
Re mark. Theorem 3 answers the question that was not considered in [5]; following Weyl, this book deals with "nonoriented" volumes only.

Since $H_{n}\left(\mathbb{R}^{n}\right)=0$, the $n$-chain $G(t)$ bounded by $F(t)$ in $\mathbb{R}^{n}$ is well defined. This chain contains only bounded connected components of $\mathbb{R}^{n} \backslash F(t)$. Note that $G(t+r)$ is nothing but the Minkowski sum of $G(t)$ and the ball in $\mathbb{R}^{n}$ of radius $r$ with center at the origin. So the following statement is similar to the fact that the mixed volume $P(a, b)$ of $a M_{1}+b M_{2}$ is a polynomial in $a, b$, where $M_{1}, M_{2}$ are convex bodies. Let us denote by $s(t)$ the volume of $G(t)$.

COROLlaRY. The oriented volume $s(t)$ bounded by a closed front $F(t) \subset \mathbb{R}^{n}$ is a polynomial in $t$ of degree $n$.

Pr o of. This immediately follows from the relation $s^{\prime}(t)=c(t)$.

Under certain restrictions, similar theorems hold for the Lobachevskii space $H^{n}$ as well.

Definition. A closed wave front in $H^{n}$ is said to be reversible if all the main curvatures radii are finite at any its point.

ExAMPLES. If geodesic lines that are normal to a front in neighbor points are hyperparallels, then the front is not reversible. A sphere is a reversible front in $H^{n}$.

For a reversible wave front $F$ in $H^{n}$, the $n$-chain bounded by $F$ is well defined. By $s(t)$ denote the volume of this chain. The proof of the following theorem is similar to that of Theorem 2.

THEOREM 4. For a reversible front in $H^{n}$, the functions $c(t)$ and $s(t)$ are polynomials in $\cosh t$ and $\sinh t$ of degree $n-1$.

2. An integral formula generalizing the Pascal law. An extra pressure inside of a ball does not move it, because the sum of all attached forces is equal to zero, as well as the sum of their angular momenta. In this section we obtain a formula expressing this principle for an isometric action of a Lie group on a Riemannian manifold. We also give some corollaries of this principle for fronts in $\mathbb{R}^{2}$ and $S^{2}$.

Consider in $\mathbb{R}^{3}$ a surface $F$ that is a boundary of a domain $U$. Suppose that $F$ is made from a thin rigid material and that there is an extra pressure of air $p$ inside of $F$, that is, in $U$. Then any element $d \sigma$ of the surface undergoes a force $f(x)$, where $x \in F$. It is clear that these forces produce no translational motion of $F$, that is, their sum is equal to zero: $\int_{F} f(x) d \sigma=0$. According to the Pascal law, the vector function $f(x)$ is equal to $p \mathbf{n}(x)$, where $\mathbf{n}$ is a unit outer normal vector, because the pressure in a liquid or a gas depends on neither point nor direction. Thus we have

$$
\int_{F} \mathbf{n} d \sigma=0 .
$$

Of course, relation (5) holds in Euclidean space of arbitrary dimension. Otherwise, a perpetual motion in $\mathbb{R}^{n}$ for a certain $n$ were possible, which were an extremely surprising thing for physicists. 
Below we prove a relation similar to (5) in quite a more general case, namely, for an action of a Lie group on a Riemannian manifold. (In the example above, the group $\mathbb{R}^{n}$ acts on itself by translations; relation (5) disregards the fact that $F$ is not forced to rotate and only shows that there is no translational movement. To deal also with a possible rotational movement, we should consider the action on $\mathbb{R}^{n}$ of the whole group $E(n)$ of orientation preserving isometries.) Now let us study some consequences of relation (5) in the case of $\mathbb{R}^{2}$.

In this case, (5) follows directly from the relation

$$
\int_{F} \mathbf{v} d l=0
$$

because $\mathbf{n}=-I \mathbf{v}$, where $I$ is the counterclockwise rotation by $90^{\circ}$ and $\mathbf{v}$ is a unit tangent vector that endows $F$ with an orientation compatible with the orientation of $U$ (that is, $F$ is counterclockwise oriented by $\mathbf{v}$ ); finally, $d l$ (rather than $d \sigma$ ) stands for the element of the arc length. Relation (6) holds if and only if the curve $F$ is closed. For a nonclosed curve $F$ starting at $A$ and ending at $B$, we have

$$
\int_{F} \mathbf{v} d l=\overrightarrow{A B} \quad \text { and } \quad \int_{F} \mathbf{n} d l=-I \overrightarrow{A B} ;
$$

this relation does not depend on the choice of a curve $F$ connecting $A$ and $B$.

Now suppose that $F$ is a cooriented wave front on a plane, that is, a closed curve with even number of semicubic singularities (cusps), and $\mathbf{n}$ is a unit normal vector defining a coorientation. In the neighborhood of a cuspidal point, the normal form of a front is $y^{2}=x^{3}$. If in this case $\mathbf{n}=(0,1)$ at the origin $(0,0) \in F$, then we say that the cuspidal point is right; otherwise, if $\mathbf{n}=(0,-1)$, then $(0,0)$ is a left cuspidal point of $F$.

It is easy to see that right and left cuspidal points alternate along $F$; in particular, the numbers of right and left cusps are equal. Let $A_{1}, \ldots, A_{k}$ be all left cuspidal points of $F$, whereas $B_{1}, \ldots, B_{k}$ be all right cuspidal points.

THEOREM 5. The following relation holds:

$$
\oint_{F} \mathbf{n} d l=-2 I\left(\sum_{i=1}^{k} B_{i}-\sum_{i=1}^{k} A_{i}\right) .
$$

The sum in the right-hand side of $(8)$ is equal to $\sum_{i} \overrightarrow{O B_{i}}-\sum_{i} \overrightarrow{O A_{i}}$; the result is independent of the choice of an auxiliary point $O$.

Proof. Consider the integral along an arc $A_{i} B_{i}$. Relations (7) directly imply that $\int_{A_{i} B_{i}} \mathbf{n} d l=-I\left(B_{i}-A_{i}\right)$. The factor 2 arises in (8) because of the arcs of the form $B_{i} A_{i+1}$. The theorem is proved.

Now consider the case of a wave front on the sphere. The same reasons on impossibility of a perpetual motion in the space $\mathbb{R}^{2}$ suggest that relation (5) should remain true. But 
what is the correct definition of the integral $\oint_{F} \mathbf{n} d l$ in this situation, where there is no well defined way to transport all vectors $\mathbf{n}(x)$ tangent to $S^{2}$ to a certain basepoint?

From the classical mechanics point of view, the problem is as follows. The standard sphere $x^{2}+y^{2}+z^{2}=1$ is fixed at the origin in $\mathbb{R}^{3}$. A field of forces $\mathbf{n}(x)$ acts on $S^{2}$ along $F$. Then the motion of the sphere is defined by the integral of the angular momentum of $\mathbf{n}(x)$ with respect to the origin, which is equal to $\oint_{F} \mathbf{k}(x) d l$, where $\mathbf{k}(x)=[\mathbf{r}(x), \mathbf{n}(x)]$ is the angular momentum of the force $\mathbf{n}(x)$ attached at a point $x$ with radius vector $\mathbf{r}(x)$. Note that the motion of the sphere is represented by a curve in the (noncommutative) Lie group $\mathrm{SO}(3)$, the velocity of this motion is an element of the tangent algebra $\mathfrak{g}=\mathrm{so}(3)$, while the angular momentum lies in $\mathfrak{g}^{*}$, i.e., is a linear function on $\mathfrak{g}$ (see [3]). Let $\mathbf{v}(x)$ be the unit vector tangent to $F$ at $x$. Then $\mathbf{k}(x)=[\mathbf{r}(x), \mathbf{n}(x)]= \pm \mathbf{v}(x)$, where the sign depends on the orientation of $v$. Thus we have the following statement.

TheOREM 6. Let $F \subset S^{2}$ be a cooriented wave front with left cuspidal points $A_{1}, \ldots, A_{k}$ and right cuspidal points $B_{1}, \ldots, B_{k}$. Then

$$
\mathbf{K}=\oint_{F}[\mathbf{r}, \mathbf{n}] d l=2\left(\sum_{i=1}^{k} B_{i}-\sum_{i=1}^{k} A_{i}\right) .
$$

In particular, $\mathbf{K}=0$ for an immersed curve $F$.

Remark. Relation (5) (which holds for immersions of $F$ to $\mathbb{R}^{n}$ ) implies that for a wave front $F$ in $\mathbb{R}^{n}$, the value of the integral $\int_{F} \mathbf{n} d \sigma$ depends on coorientation of $F$ and on the set $F \backslash F_{\text {reg }}$ of singular points of $F$ that has codimension 1 (singular sets of higher codimension do not affect the integral) but is independent of the regular part $F_{\text {reg }}$ of $F$.

Finally, let us consider the general case. Suppose that a Lie group $G$ with the Lie algebra $\mathfrak{g}$ acts isometrically on an oriented Riemannian manifold $M^{n}$. Let $F \subset M$ be an immersed connected cooriented hypersurface that bounds a domain $U \subset M$ (or, at least, is homological to zero over $\mathbb{Q}$; so, if $H_{n-1}\left(M^{n}, \mathbb{Q}\right)=0$, then we can take any closed oriented hypersurface). Let $\mathbf{n}$ be a unit normal vector field defining a coorientation of $F$. Suppose that a field of forces $\mathbf{n}(x)$ acts on the rigid body $U$, where $\partial U=c F, c \in \mathbb{Q} \backslash\{0\}$.

Definition. The angular momentum $\mathbf{k}(x)$ is the linear function on $\mathfrak{g}$ that takes a vector $\tau \in \mathfrak{g}$ to the inner product $\left(\mathbf{n}(x), \mathbf{q}_{\tau}(x)\right)$, where $\mathbf{q}_{\tau}$ is a vector field on $M$ related to the generator $\tau$. More precisely, if $g(t) \subset G$ is a one-parameter subgroup such that $\left.\frac{d}{d t} g(t)\right|_{t=0}=\tau$, then $\mathbf{q}_{\tau}(x)=\left.\frac{d}{d t}(g(t))(x)\right|_{t=0}$.

THEOREM 7. If the assumptions stated above hold, then the integral angular momentum $\mathbf{K} \in \mathfrak{g}^{*}$ of the forces $\mathbf{n}(x)$ is equal to zero:

$$
\mathbf{K}=\int_{F} \mathbf{k}(x) d \sigma=0 .
$$

Here the area element $d \sigma$ is induced by the first fundamental form of $F$. 
Proof. For any $\tau \in \mathfrak{g}$, we have

$$
\begin{aligned}
\mathbf{K}(\tau)=\int_{F}(\mathbf{k}(x))(\tau) d \sigma=\int_{F}(\mathbf{n}(x) & \left., \mathbf{q}_{\tau}(x)\right) d \sigma \\
& =c^{-1} \int_{c F}\left(\mathbf{n}(x), \mathbf{q}_{\tau}(x)\right) d \sigma=c^{-1} \int_{U} \operatorname{div} \mathbf{q}_{\tau}=0 .
\end{aligned}
$$

In the last but one equality we use the Stokes formula, and the last equality holds because the divergence of the field $\mathbf{q}_{\tau}$ equals zero. In fact, the flow of $\mathbf{q}_{\tau}$ acts on $M$ by isometries $g(t) \in G$, and thus preserves the volume form. Therefore, the covector $\mathbf{K} \in \mathfrak{g}^{*}$ is equal to zero. The theorem is proved.

Remark (A. O. Viro). The proof of Theorem 7 only uses the fact that the action of $G$ on $M$ preserves the volume (rather than the metric). However, a metric on $M$ is involved in definitions of $\mathbf{n}(x)$ and $\mathbf{k}(x)$.

\section{References}

[1] S. S. Anisov, The "area-length" duality and the characteristic 2-chain (in Russian), Mat. Zametki 58:3 (1995), 445-446; English transl.: Math. Notes 58 (1995), 983-984.

[2] V. I. Arnol'd, Singularities of ray systems (in Russian), Uspekhi Mat. Nauk 38:2 (1983), 77-147; English transl.: Russian Math. Surveys 38:2 (1983), 87-176.

[3] V. I. Arnol'd, Mathematical Methods of Classical Mechanics, second ed., Springer, New York, 1989.

[4] V. I. Arnol'd, The geometry of spherical curves and the algebra of quaternions (in Russian), Uspekhi Mat. Nauk 50:1 (1995), 3-68; English transl.: Russian Math. Surveys 50:1 (1995), 1-68.

[5] A. Gray, Tubes, Addison-Wesley, Redwood City, 1990.

[6] J. Milnor, Morse Theory, Ann. of Math. Stud. 51, Princeton Univ. Press, Princeton, 1963. 